\title{
Ball lightning plasma and plasma arc formation during the microwave heating of carbons
}

\author{
J. A. Menéndez*, E. J. Juárez-Pérez, E. Ruisánchez, J. Bermúdez, A. Arenillas \\ Instituto Nacional del Carbón, CSIC, Apartado 73, Oviedo 33080, Spain
}

\begin{abstract}
Microwave heating can be used to promote heterogeneous reactions with carbon materials, mainly because of the heating mechanism involved in microwave radiation and the phenomenon known as microplasmas. This work presents photographic evidence of plasma formation when different carbon materials are subjected to microwave heating. Two different kinds of plasmas were observed: ball lightning and arc discharge plasmas. The intensity of the plasmas in the less ordered carbon was significantly higher at the beginning of the process.
\end{abstract}

Microwave heating of carbons is used in processes such as the activation or regeneration of activated carbons, gas-phase chemical reactions, pyrolysis, carbonization, the synthesis of carbon gels, the generation of carbon nanofilaments, etc. [1]. In general, it has been reported that the use of microwave heating offers certain advantages over conventional heating. In most cases the process can be carried out in a much shorter time (e.g. pyrolysis, activation). Sometimes the products obtained are different or have different properties (e.g. pyrolysis, catalytic reactions, synthesis of carbons). Generally the differences between the microwave and conventional heating of carbon materials are attributed to the different heating mechanisms (dielectric heating vs. convection and conduction) and, especially, to the occurrence of a phenomenon referred to as "hot spots". When direct observation of the carbon bed during microwave heating is possible, it can be observed that small sparks or electric arcs are produced. These sparks are confined to a very tiny space and last only a fraction of a second. Hence they are often referred to as small localized plasmas or microplasmas $[2,3,4]$. However, there are no clear evidences that genuine plasmas are formed. The aim of this communication is to present photographic evidence that, at *Corresponding author. Fax: +34 9852976 62. E-mail address: angelmd@incar.csic.es (J.A. Menéndez) 
least, two different types of plasmas can appear when carbon materials are subjected to microwave heating, gaining some insight into the characteristics of such plasmas.

The experimental device used was a multimode cavity microwave oven equipped with a stainless steel ungrounded K-type thermocouple that not only allows the temperature of the sample to be measured, but is also connected to a PID controller so that the heating rate and final temperature can be programmed. A sample of approximately $3 \mathrm{~g}$ of carbon was placed on an alumina tray, which in turn was placed inside a $30 \mathrm{~mm}$ diameter quartz tube supported by a quartz wool layer. The tube was closed at both ends with perforated rubber stoppers that allowed a flow of $\mathrm{N}_{2}(360$ $\mathrm{mL} / \mathrm{min}$ ) to pass through the quartz tube, thus enabling an inert atmosphere to be maintained during the experiments. All the materials used were practically transparent to microwaves, with the exception of the thermocouple which reflected the microwaves and the carbon that absorbed them. This experimental set-up is shown in the photograph of Figure 1. The experiments consisted in setting the final temperature at $700{ }^{\circ} \mathrm{C}$ and switching on the microwave at $50 \%$ maximum power (i.e., on/off pulses of $700 \mathrm{~W}$ of approximately $1 \mathrm{~s}$. duration). The experiment was video recorded through a window in the microwave door using a Cannon Ixus100 IS, HD $1280 \times 720,30$ fps. Selected frames of the recorded films are shown in the figures. The experiments were carried out on five different types of carbon which were ranked from the most ordered to the least ordered structures, according to their graphitic character, as follows: (i) graphite, (ii) metallurgical coke, (iii) anthracite, (iv) activated carbon and (v) a char from the pyrolysis of biomass. In all cases the carbon grains ranged from 0.5 to $3 \mathrm{~mm}$. 


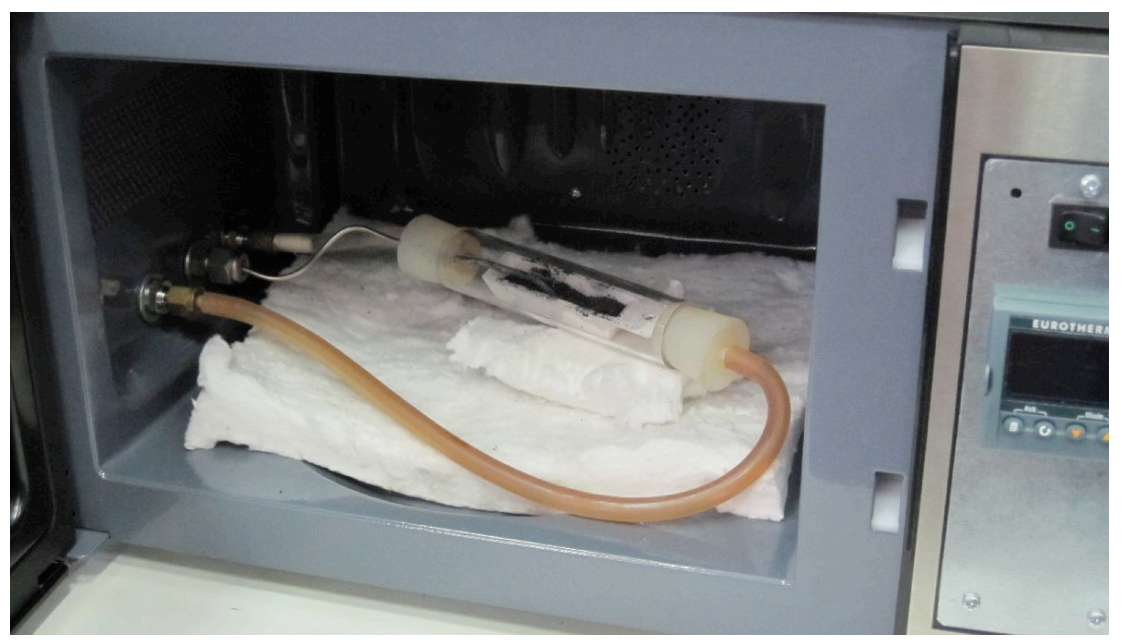

Figure 1. Experimental set-up.

In general, after 1 or 2 seconds of irradiation with microwaves, tiny flashes of light were observed to spread across the whole surface of the carbon bed, and also inside the carbon bed itself (this can be seen through the walls of the alumina tray, as in last picture of Figure 2). Most of these flashes appeared to be no longer than $1 \mathrm{~mm}$ and to last only a fraction of a second. Occasionally longer flashes of about $10-30 \mathrm{~mm}$ were observed as well. This phenomenon is illustrated in the video as supporting material. At the beginning of the experiment the flashes of light were concentrated in certain regions of the carbon bed. Although the microplasmas occurred chiefly around the tip of the thermocouple, other regions (arbitrarily located depending on the experiment) were also prone to such occurrences, independently of the experiment performed. Moreover, in a number of experiments carried out without the use of a thermocouple, randomly located microplasmas were observed as well. The temperature in these regions increased more rapidly than in the rest of the carbon bed, i.e., such regions became incandescent before the rest. The K-thermocouple is not a meaningful probe for the temperature of the plasma, since the temperature in plasmas is a very complex issue, as different species may be at different temperatures. The systems are not in thermal equilibrium and two temperatures can exits at the same place and time [4]. As the microwave radiation proceeded the entire carbon bed became incandescent and, the average temperature (i.e., the temperature as measured by the 
thermocouple) was similar in all parts of the bed. At this point, the frequency of occurrence and intensity of the microplasmas seemed to relapse. However this could be a false impression since in an incandescent carbon bed tiny plasmas are more difficult to be perceived. Nevertheless, these plasmas are still relatively abundant as illustrated in Figure 3.
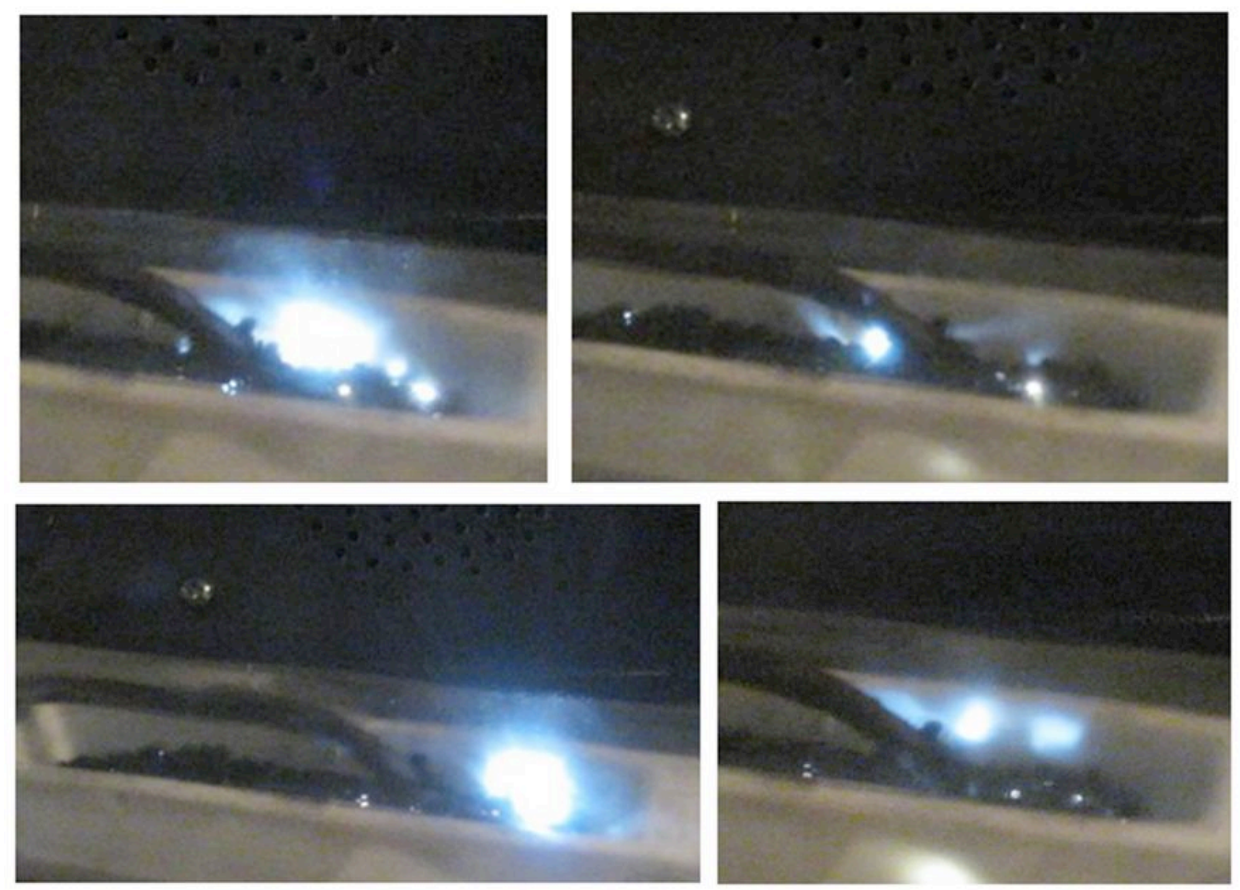

Figure 2. Ball lightning plasma formation at the initial stages of the experiment, (average temperature as measured by a thermocouple: $<400{ }^{\circ} \mathrm{C}$ ). The experiment was carried out over activated carbon and in the presence of a thermocouple.

The microplasmas observed can be divided into two different types. Quasi-spherical plasmas, like those shown in Figure 2 and in the two top pictures of Figure 3 and electric arcs like those in the two bottom pictures. The spherical plasmas are examples of the formation, at laboratory scale, of the "unusual" and "controversial" ball lightning plasmas $[5,6]$. Ever since the earliest attempt to explain ball lightning plasmas made by Nikola Tesla in $1904^{*}$ to the intriguing black hole hypothesis proposed by Rabinowitz [7] who suggested that small quiescent black holes are the core energy source of ball lightning plasmas, various theories have been put forward, but there is 
no widely-accepted explanation for the phenomenon [5]. These ball lightning plasmas are more abundant at the beginning of the experiments when the temperature of the carbon bed is still relatively low $\left(<400^{\circ} \mathrm{C}\right)$. On the other hand, electric arcs (or arc discharges), like those shown in the two bottom pictures of Figure 3 appear at higher temperatures $\left(400-700{ }^{\circ} \mathrm{C}\right)$. It should be mentioned that an electric arc is an electrical breakdown of a gas which produces an ongoing plasma discharge, resulting from a current flowing through normally non-conductive media such as air. These two types of plasma are very different not only as regards their shape, as can be seen in Figures 2 and 3, but also, as mentioned above, as regards their nature.
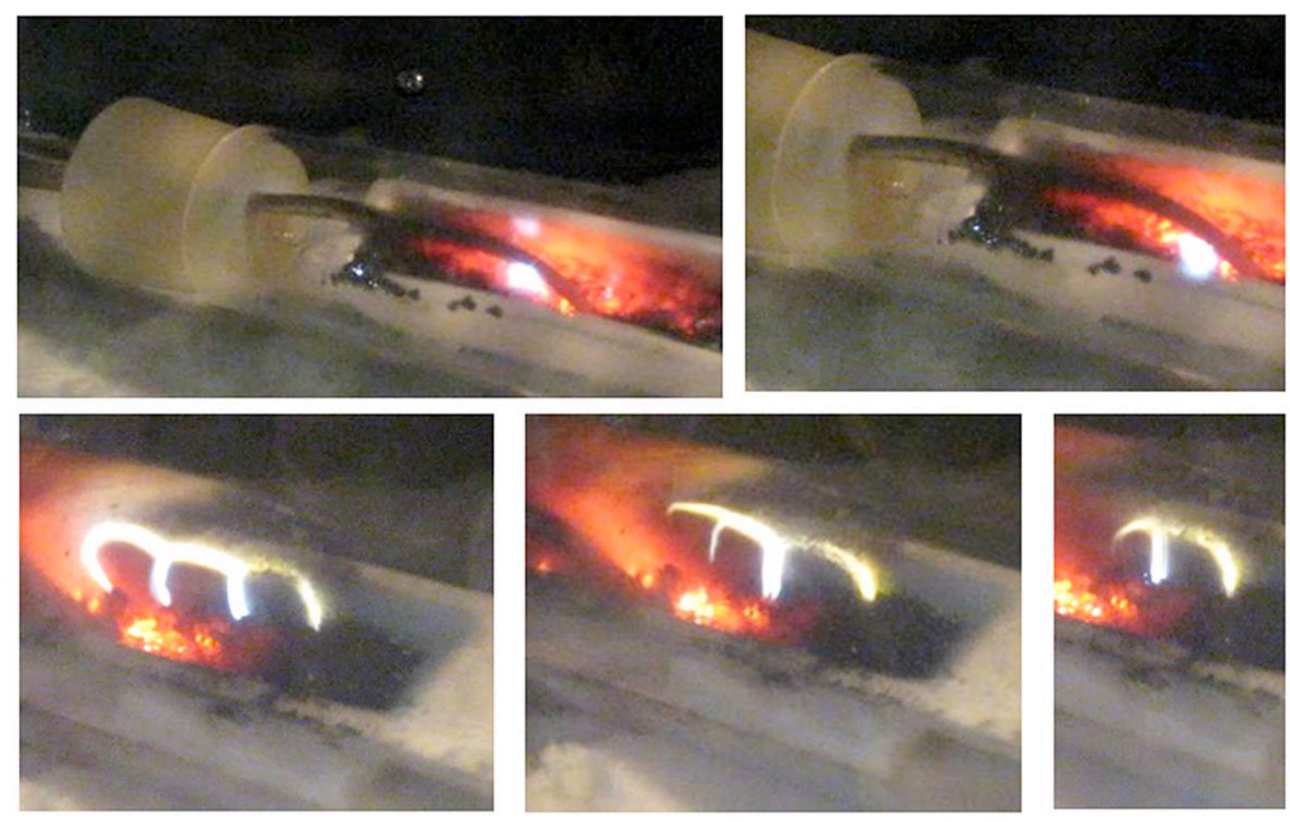

Figure 3. Ball lightning plasmas and plasma arcs after one minute of irradiation (average temperature measured with the thermocouple: $400-700^{\circ} \mathrm{C}$ ). The experiment was carried out over activated carbon and in the presence of a thermocouple.

All types of carbon, when subjected to the microwave heating, present a more or less similar behavior to that described above. However, it is known that different carbons heat to different extents in a microwave field depending on their structure and composition [1]. Thus, it was observed that, at the beginning of the experiment, before the carbon bed became incandescent, the intensity and size of the ball plasmas were higher in the less ordered carbons, i.e., the 
activated carbon and the biomass char. In these two carbons some of the ball plasmas reached a size of $10-30 \mathrm{~mm}$. Surprisingly, in the case of the biomass char some of the ball lightning plasmas were observed to extend beyond the walls of the tube without apparently experiencing any alteration or obstruction (see Figure 4). Possible overexposure of the camera sensor cannot be discarded as the cause of this effect. However, as can be seen in Figure 4 (top) most of the large plasmas recorded with the camera appeared to be confined inside the quartz tube, plasmas like the one in the bottom picture of Figure 4 being the exception rather than the rule. If confirmed, this phenomenon reported is extremely unusual since plasmas cannot penetrate through glass. However, an explanation of how ball lighting might be able to do so is given by Torchigin [8].
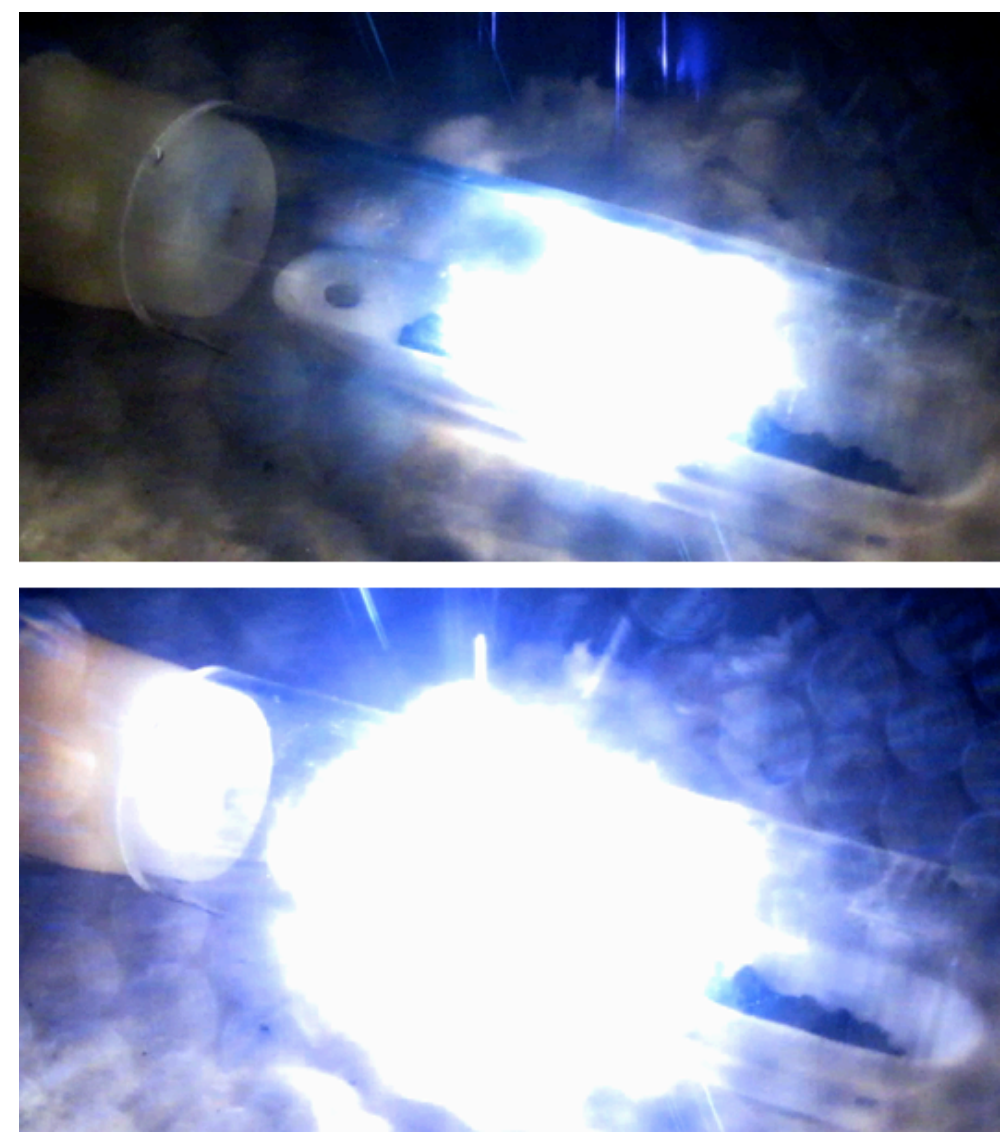

Figure 4. Ball lightning plasma formation at the initial stage of the experiment. While in the top picture the plasma appears to be confined inside the quartz tube, the plasma shown in the picture underneath appears to extend beyond the walls of the tube. The experiment was carried out over biomass char and in the absence of a thermocouple. 
In sum, in the conditions described in this work the microwave heating of carbon materials was observed to give rise to the formation of two different kinds of plasma i.e., ball lightning or arc discharge plasmas.

\section{Acknowledgements}

The financial support from the PCTI-Asturias (Projects PEST08-03 and PEST08-21) and the Carburos Metálicos-Air Products Group, under Project CEN-2008-1027, a CENIT Project belonging to the Program Ingenio 2010, of the Spanish Government (CDTI), is acknowledged.

\section{References}

1. Menéndez JA, Arenillas A, Fidalgo B, Fernández Y, Zubizarreta L, Calvo EG, Bermúdez JM. Microwave heating processes involving carbon materials. Fuel Processing Technology 2010; $91: 1-8$

2. Dawson EA, Parkes GMB, Barnes PA, Bond G, Mao R. The generation of microwaveinduced plasma in granular activated carbon under fluidized bed conditions. Carbon 2008; 46:220-28.

3. Fernández Y, Fidalgo B, Domínguez A, Arenillas A, Menéndez JA. Carbon nanofilament synthesis by the decomposition of $\mathrm{CH}_{4} / \mathrm{CO}_{2}$ under microwave heating. Carbon 2007; 45:1706-9.

4. Phillips, J., Luhrs, C. C., Richard, M. Engineering Particles Using the AerosolThrough-Plasma Method. IEEE Transactions on Plasmas 2009; 37: 726-39.

5. Smirnov M. The properties and the nature of ball lightning. Physics Reports 1987; 152(4):177-226.

6. Ohtsuki YH, Ofuruton H. Plasma fireballs formed by microwave interference in air. Nature 1991; 350:139-41. 
7. Rabinowitz M. Little Black Holes: Dark Matter and Ball Lightning. Astrophysics and Space Science 1998; 262(4):391-410.

8. Torchigin VP. On the Nature of Ball Lightning. Doklady Physics 2003; 48(3):108-11. 